\title{
Multisource Images Analysis Using Collaborative Clustering
}

\author{
Germain Forestier, Cédric Wemmert, and Pierre Gançarski \\ LSIIT, UMR 7005 CNRS/ULP, University Louis Pasteur, 67070 Strasbourg Cedex, France \\ Correspondence should be addressed to Germain Forestier, forestier@lsiit.u-strasbg.fr \\ Received 1 October 2007; Revised 20 February 2008; Accepted 26 February 2008 \\ Recommended by C. Charrier
}

The development of very high-resolution (VHR) satellite imagery has produced a huge amount of data. The multiplication of satellites which embed different types of sensors provides a lot of heterogeneous images. Consequently, the image analyst has often many different images available, representing the same area of the Earth surface. These images can be from different dates, produced by different sensors, or even at different resolutions. The lack of machine learning tools using all these representations in an overall process constraints to a sequential analysis of these various images. In order to use all the information available simultaneously, we propose a framework where different algorithms can use different views of the scene. Each one works on a different remotely sensed image and, thus, produces different and useful information. These algorithms work together in a collaborative way through an automatic and mutual refinement of their results, so that all the results have almost the same number of clusters, which are statistically similar. Finally, a unique result is produced, representing a consensus among the information obtained by each clustering method on its own image. The unified result and the complementarity of the single results (i.e., the agreement between the clustering methods as well as the disagreement) lead to a better understanding of the scene. The experiments carried out on multispectral remote sensing images have shown that this method is efficient to extract relevant information and to improve the scene understanding.

Copyright (C) 2008 Germain Forestier et al. This is an open access article distributed under the Creative Commons Attribution License, which permits unrestricted use, distribution, and reproduction in any medium, provided the original work is properly cited.

\section{INTRODUCTION}

Unsupervised classification, also called clustering, is a wellknown machine learning tool which extracts knowledge from datasets $[1,2]$. The purpose of clustering is to group similar objects into subsets (called clusters), maximizing the intracluster similarity and the intercluster dissimilarity. Many clustering algorithms have been developed during the last 40 years,each one is based on a different strategy. In image processing, clustering algorithms are usually used by considering the pixels of the image as data objects: each pixel is assigned to a cluster by the clustering algorithm. Then, a map is produced, representing each pixel with the colour of the cluster it has been assigned to. This cluster map, depicting the spatial distribution of the clusters, is then interpreted by the expert who assigns to each cluster (i.e., colour in the image) a mean in terms of thematic classes (vegetation, water, etc.).

In contrast to the supervised classification, unsupervised classification requires very few inputs. The classification process only uses spectral properties to group pixels together. However, it requires a precise parametrization by the user because the classification is performed without any control.

Other potential problems exist, especially when the user attempts to assign a thematic class to each produced cluster. On the one hand, some thematic classes may be represented by a mix of different types of surface covers: a single thematic class may be split among two or more clusters (e.g., a park is often an aggregate of vegetation, sand, water, etc.). On the other hand, some of the clusters may be meaningless, as they include too many mixed pixels: a mixed pixel (mixel) represents the average energy reflected by several types of surface present within the studied area.

These problems have increased with the recent availability of very high-resolution satellite sensors, which provide many details of the land cover. Moreover, several images with different characteristics are often available for the same area: different dates, from different kinds of remote sensing acquisition systems (i.e., with different numbers of sensors and wavelengths) or different resolutions (i.e., different sizes 
of surface of the area that a pixel represents on the ground). Consequently, the expert is confronted to a too great mass of data: the use of classical knowledge extraction techniques became too complex. It needs specific tools to extract efficiently the knowledge stored in each of the available images.

To avoid the independent analysis of each image, we propose to use different clustering methods, each working on a different image of the same area. These different clustering methods collaborate together during a refinement step of their results, to converge towards a similar result. At the end of this collaborative process, the different results are combined using a voting algorithm. This unified result represents a consensus among all the knowledge extracted from the different sources. Furthermore, the voting algorithm highlights the agreement and the disagreement between the clustering methods. These two pieces of information, as well as the result produced by each clustering method, lead to a better understanding of the scene by the expert.

The paper is organized as follows. First, an overview of multisource applications is introduced in Section 2. The collaborative method to combine different clustering algorithms is then presented in Section 3. Section 4 presents in details the paradigm of multisource images and the different ways to use it in the collaborative system. Section 5 shows an experimental evaluation of the developed methods, and finally, conclusions are drawn in Section 6.

\section{MULTISOURCE IMAGES ANALYSIS}

In the domain of Earth observation, many works focus on the development of data-fusion techniques to take advantage of all the available data on the studied area. As discussed in [3], multisource image analysis can be achieved at different levels, according to the stage where the fusion takes place: pixel, feature, or decision level.

At pixel level, data fusion consists in creating a fused image based on the sensors measurements by merging the values given by the various sources. A method is proposed in [4] for combining multispectral, panchromatic, and radar images by using conjointly the intensity-hue-saturation transform and the redundant wavelet decomposition. In [5], the authors propose a multisource data-fusion mechanism using generalized positive Boolean functions which consists of two steps: a band generation is carried out followed by a classification using a positive Boolean function-based classifier. In the case of feature fusion, the first step creates new features from the various datasets; these new features are merged and analyzed in a second step. For example, a segmentation can be performed on the different image sources and these segmentations are fused [6]. In [7], the authors present another method based on the DempsterShafer theory of evidence and using the fuzzy statistical estimation maximization (FSEM) algorithm to find an optimal estimation of the inaccuracy and uncertainty of the classification.

The fusion of decisions consists in finding a single decision (also called consensus) from all the decisions produced by the classifiers. In [8], the authors propose a method based on the combination of neural networks for multisource classification. The system exposed in [9] is composed of an ensemble of classifiers trained in a supervised way on a specific image, and can be retrained in an unsupervised way to be able to classify a new image. In [10], a general framework is presented for combining information from several supervised classifiers using a fuzzy decision rule.

In our work, we focus on fusion of decisions from unsupervised classifications, each one produced from a different image. Contrary to the methods presented above, we propose a mechanism which finds a consensus according to the decisions taken by each of the unsupervised classifier.

\section{COLLABORATIVE CLUSTERING}

Many works focus on combining different results of clustering, which is commonly called clustering aggregation [11], multiple clusterings [12], or cluster ensembles [13, 14]. All these approaches try to combine different results of clustering in a final step. In fact, these results must have the same number of clusters (vote-based methods) [14] or the expected clusters must be separable in the data space (coassociation-based methods) [12]. This latter property is almost never encountered in remote sensing image analysis. It is difficult to compute a consensual result from clustering results with different numbers of classes or different structures (flat partitioning or hierarchical result) because of the lack of a trivial correspondence between the clusters of these different results. To address the problem, we present in this section a framework where different clustering methods work together in a collaborative way to find an agreement about their proposals. This collaborative process consists in an automatic and mutual refinement of the clustering results, until all the results have almost the same number of clusters, and all the clusters are statistically similar. At the end of this process, as the results have comparable structures, it is possible to define a correspondence function between the clusters, and to apply a unifying technique such as a voting method [15].

Before the description of the collaborative method, we introduce the correspondence function used within it.

\subsection{Intercluster correspondence function}

There is no problem to associate classes of different supervised classifications as a common set of class labels is given for all the classifications. Unfortunately, in the case of unsupervised classifications, the results may not have a same number of clusters, and no information is available about the correspondence between the different clusters of the different results.

To address the problem, we have defined a new intercluster correspondence function, which associates to each cluster from a result, a cluster from each of the other results.

Let $\left\{\mathcal{R}^{i}\right\}_{1 \leq i \leq m}$ be the set of results given by the different algorithms. Let $\left\{\mathcal{C}_{k}^{i}\right\}_{1 \leq k \leq n_{i}}$ be the clusters of the result $\mathcal{R}^{i}$. Figure 1 shows an example of such results. 

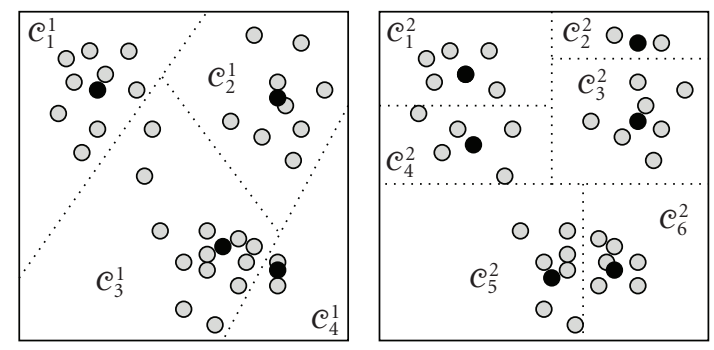

Figure 1: Two clustering results of the same data but using a different method.

The corresponding cluster $\mathrm{CC}\left(\mathcal{C}_{k}^{i}, \mathcal{R}^{j}\right)$ of a cluster $\mathcal{C}_{k}^{i}$ from $\mathcal{R}^{i}$ in the result $\mathcal{R}^{j}, i \neq j$, is the cluster from $\mathcal{R}^{j}$ which is the most similar to $\mathcal{C}_{k}^{i}$ :

$$
\begin{aligned}
& \operatorname{CC}\left(\mathcal{C}_{k}^{i}, \mathcal{R}^{j}\right)=\mathcal{C}_{\ell}^{j} \\
& \text { with } S\left(\mathcal{C}_{k}^{i}, \mathcal{C}_{\ell}^{j}\right)=\max \left(\left\{S\left(\mathcal{C}_{k}^{i}, \mathcal{C}_{l}^{j}\right), \quad \forall l \in\left[1, n_{j}\right]\right\}\right),
\end{aligned}
$$

where $S$ is the intercluster similarity which evaluates the similarity between two clusters of two different results.

It is calculated from the recovery of the clusters in two steps. First, the intersection between each couple of clusters $\left(\mathcal{C}_{k}^{i}, \mathcal{C}_{l}^{j}\right)$, from two different results $\mathcal{R}^{i}$ and $\mathcal{R}^{j}$, is calculated and written in the confusion matrix $\mathcal{M}^{i, j}$ :

$$
\mathcal{M}^{i, j}=\left(\begin{array}{ccc}
\alpha_{1,1}^{i, j} & \cdots & \alpha_{1, n_{j}}^{i, j} \\
\vdots & \ddots & \vdots \\
\alpha_{n_{i}, 1}^{i, j} & \cdots & \alpha_{n_{i}, n_{j}}^{i, j}
\end{array}\right), \quad \text { where } \alpha_{k, l}^{i, j}=\frac{\left|\left\{\mathcal{C}_{k}^{i} \cap \mathcal{C}_{l}^{j}\right\}\right|}{\left|\mathcal{C}_{k}^{i}\right|}
$$

Then, the similarity $S\left(\mathcal{C}_{k}^{i}, \mathcal{C}_{l}^{j}\right)$ between two clusters $\mathcal{C}_{k}^{i}$ and $\mathcal{C}_{l}^{j}$ is evaluated by observing the relationship between the size of their intersection and the size of the cluster itself, and by taking into account the distribution of the data in the other clusters as follows:

$$
S\left(\mathcal{C}_{k}^{i}, \mathcal{C}_{l}^{j}\right)=\alpha_{k, l}^{i, j} \alpha_{l, k}^{j, i}
$$

Figure 2 presents the correspondence function obtained by using the intercluster similarity on the results shown in Figure 1 .

\subsection{Collaborative process overview}

The entire clustering process is broken down in three main following phases:

(i) initial clusterings: each clustering method computes a clustering of the data using its parameters;

(ii) results refinement: a phase of convergence of the results, which consists of conflicts evaluation and resolution, is iterated as long as the quality of the results and their similarity increase;

(iii) Unification: the refined results are unified using a voting algorithm.

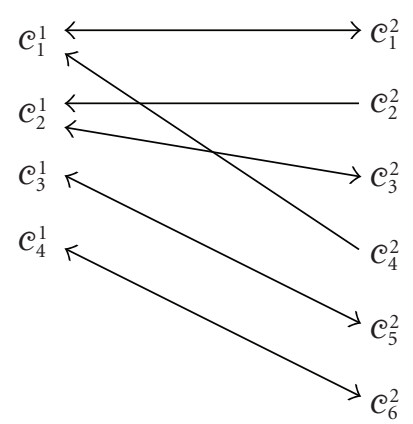

Figure 2: The correspondence between the clusters of the two results from Figure 1 using the intercluster similarity by recovery.

\subsubsection{Initial clusterings}

During the first step, each clustering method is initialized with its own parameters and a clustering is performed on a remotely sensed image: all the pixels are grouped into different clusters.

\subsubsection{Results refinement}

The mechanism we propose for refining the results is based on the concept of distributed local resolution of conflicts, by the iteration of four phases:

(i) detection of the conflicts by evaluating the dissimilarities between couples of results;

(ii) choice of the conflicts to solve;

(iii) local resolution of these conflicts;

(iv) management of the local modifications in the global result (if they are relevant).

\section{(a) Conflicts detection}

The detection of the conflicts consists in seeking all the couples $\left(\mathcal{C}_{k}^{i}, \mathcal{R}^{j}\right), i \neq j$, such as $\mathcal{C}_{k}^{i} \neq \operatorname{CC}\left(\mathcal{C}_{k}^{i}, \mathcal{R}^{j}\right)$. One conflict $\mathcal{K}_{k}^{i, j}$ is identified by one cluster $\mathcal{C}_{k}^{i}$ and one result $\mathcal{R}^{j}$.

We associate to each conflict a measurement of its importance, the conflict importance coefficient, calculated according to the intercluster similarity

$$
\mathrm{CI}\left(\mathcal{K}_{k}^{i, j}\right)=1-S\left(\mathcal{C}_{k}^{i}, C C\left(\mathcal{C}_{k}^{i}, \mathcal{R}^{j}\right)\right)
$$

\section{(b) Choice of the conflicts to solve}

During an iteration of refinement of the results, several local resolutions are performed in parallel. A conflict is selected in the set of existing conflicts and its resolution is started. This conflict, like all those concerning the two results involved in the conflict, are removed from the list of the conflicts. This process is iterated, until the list of the conflicts is empty.

Different heuristics can be used to choose the conflict to solve, according to the conflict importance coefficient (4). We choose to try to solve the most important conflict first. 


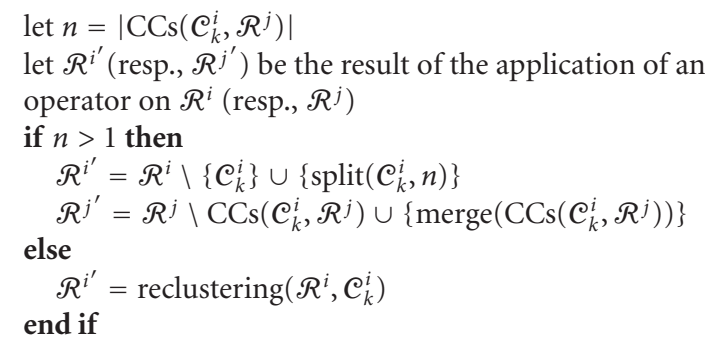

Algorithm 1

\section{(c) Local resolution of a conflict}

The local resolution of a conflict $\mathcal{K}_{k}^{i, j}$ consists of applying an operator on each result involved in the conflict, $\mathcal{R}^{i}$ and $\mathcal{R}^{j}$, to try to make them more similar.

The operators that can be applied to a result are the following:

(i) merging of clusters: some clusters are merged together (all the objects are merged in a new cluster that replaces the clusters merged),

(ii) splitting of a cluster in subclusters: a clustering is applied to the objects of a cluster to produce subclusters,

(iii) reclustering of a group of objects: one cluster is removed and its objects are reclassified in all the other existing clusters.

The operator to apply is chosen according to the corresponding clusters of the cluster involved in the conflict. The corresponding clusters (CCs) of a cluster are an extension of the definition of the corresponding cluster (1):

$$
\operatorname{CCs}\left(\mathcal{C}_{k}^{i}, \mathcal{R}^{j}\right)=\left\{\mathcal{C}_{l}^{j} \mid S\left(\mathcal{C}_{k}^{i}, \mathcal{C}_{l}^{j}\right)>p_{\mathrm{cr}}, \forall l \in\left[1, n_{j}\right]\right\},
$$

where $p_{\mathrm{cr}}, 0 \leq p_{\mathrm{cr}} \leq 1$, is given by the user. Having found the corresponding clusters of the cluster involved in the conflict, an operator is chosen and applied as shown in Algorithm.

But the application of the two operators is not always relevant. Indeed, it does not always increase the similarity of the results implied in the conflict treated, and especially, the iteration of conflict resolutions may lead to a trivial solution where all the methods are in agreement. For example, they can converge towards a result with only one cluster including all the objects to classify, or towards a result having one cluster for each object. These two solutions are not relevant and must be avoided.

So we defined a criterion $\gamma$, called local similarity criterion, to evaluate the similarity between two results, based on the intercluster similarity $S(3)$ and a quality criterion $\delta$ (given by the user):

$$
\gamma^{i, j}=\frac{1}{2}\left(p_{s} \cdot\left(\frac{1}{n_{i}} \sum_{k=1}^{n_{i}} \omega_{k}^{i, j}+\frac{1}{n_{j}} \sum_{k=1}^{n_{j}} \omega_{k}^{j, i}\right)+p_{q} \cdot\left(\delta^{i}+\delta^{j}\right)\right),
$$

where

$$
\omega_{k}^{i, j}=\sum_{l=1}^{n_{j}} S\left(\mathfrak{C}_{k}^{i}, \operatorname{CC}\left(\mathcal{C}_{k}^{i}, \mathcal{R}^{j}\right)\right)
$$

and, $p_{q}$ and $p_{s}$ are given by the user $\left(p_{q}+p_{s}=1\right)$. The quality criterion $\delta^{i}$ represents the internal quality of a result $\mathcal{R}^{i}$ (the compactness of its clusters, e.g.).

At the end of each conflict resolution, the local similarity criterion enables to choose which couple of results are to be kept: the two new results, the two old results, or one new result with one old result.

\section{(d) Global management of the local modifications}

After the resolutions of all these local conflicts, a global application of the modifications proposed by the refinement step is decided if it improves the quality of the global result. The global agreement coefficient of the results is evaluated according to all the local similarity between each couple of results. It evaluates the global similarity of the results and their quality:

$$
\boldsymbol{\Gamma}=\frac{1}{m} \sum_{i=1}^{m} \Gamma^{i}
$$

where

$$
\Gamma^{i}=\frac{1}{m-1} \sum_{\substack{j=1 \\ j \neq i}}^{m} \gamma^{i, j} .
$$

Even if the local modifications decrease this global agreement coefficient, the solution is accepted to avoid to fall in a local maximum. If the coefficient is decreasing too much, all the results are reinitialized to the best temporary solution (the one with the best global agreement coefficient).

The global process is iterated until some conflicts can be solved.

\subsubsection{Unification}

In the final step, all the results tend to have the same number of clusters, which are increasingly similar. Thus, we use a voting algorithm [15] to compute a unified result combining the different results. This multiview-voting algorithm enables to combine in one unique result, many different clustering results that have not necessarily the same number of clusters.

The basic idea is that for each object to cluster, each result $\mathcal{R}^{i}$ votes for the cluster it has found for this object, $\mathcal{C}_{k}^{i}$ for example, and for the corresponding cluster of $\mathcal{C}_{k}^{i}$ in all the other results. The maximum of these values indicates the best cluster for the object, for example $\mathcal{C}_{l}^{j}$. This means that this object should be in the cluster $\mathcal{C}_{l}^{j}$ according to the opinion of all the methods.

After having done the vote for all objects, a new cluster is created for each best cluster found if a majority of the methods has voted for this cluster. If not, the object is affected to a special cluster, containing all the objects that do not have the majority, which means they have been classified differently in too many results. 


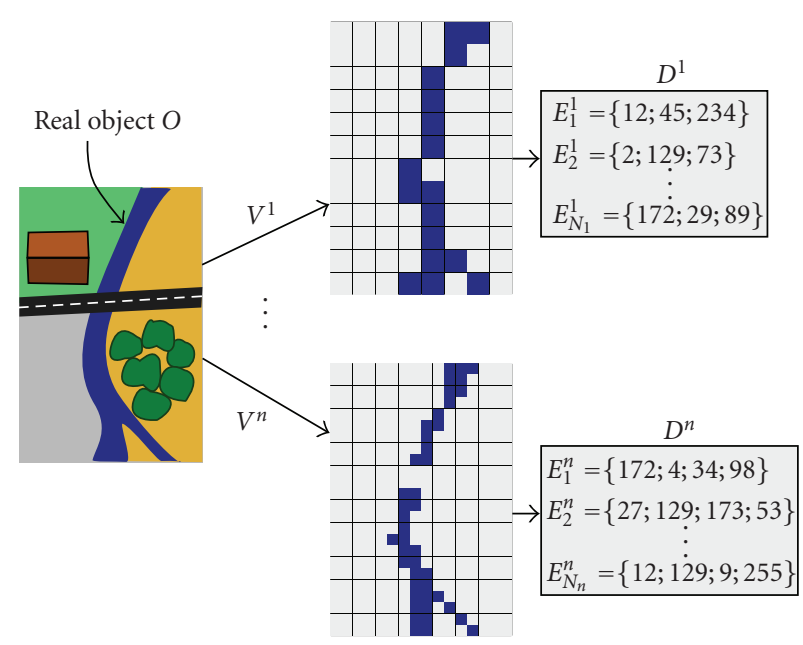

Figure 3: Different points of view $V^{1}$ to $V^{n}$ on a same object $O$ (the river) producing different descriptions $D^{1}$ to $D^{n}$ of the object.

\section{MULTISOURCE IMAGE PARADIGM}

The method described in the previous section can use different types of clustering algorithms, but they work with only one common dataset (i.e., the same image for each clustering algorithm). In this section, we describe how we make the collaborative method able to combine different sources of data and to extract knowledge from them.

The problem can be described as follows. There exists one real object $O$ that can be viewed from different points of view, and the goal is to find one description of this object, according to all the different points of view (Figure 3). Each view $V^{i}$ of the object is represented by a data set $D^{i}$ which is composed of many elements $\left\{E_{1}^{i}, \ldots, E_{N_{i}}^{i}\right\}$. Each element $E_{k}^{i}$ is described by a set of attributes $\left\{\left(a_{l}^{i, k}, v_{l}^{i, k}\right)\right\}_{1<l<n_{i, k}}$ composed of a name $a$ and a value $v$.

Three different cases can be happened (Figure 4):

(a) $E_{k}^{i}=E_{k}^{j}$ for all $i, j, a_{l}^{i, k}=a_{l}^{j, k}$ for all $l$ and $v_{l}^{i, k} \neq v_{l}^{j, k}$ (e.g., two remote sensing images of a same region, from the same satellite, but at different seasons);

(b) $E_{k}^{i}=E_{k}^{j}$ for all $i, j$ and $a_{l}^{i, k} \neq a_{l}^{j, k}$ (e.g., two remote sensing images of a same region, having a same resolution, but from two different satellites with different sensors);

(c) $E_{k}^{i} \neq E_{k}^{j}$ for all $i, j \mid i \neq j$ (e.g., two remote sensing images of a same region, but having a different resolution, and from two different satellites with different sensors).

\subsection{Multisource objects clustering}

A first method to classify multisource objects is to merge the attributes from the different sources. Each object has a new description composed of the attributes of all the sources (Figure 5(a)). But this technique may produce many clusters because the description of the object would be too precise (i.e., would have an important number of attributes). So it is hard to discriminate the objects. Indeed, due to the

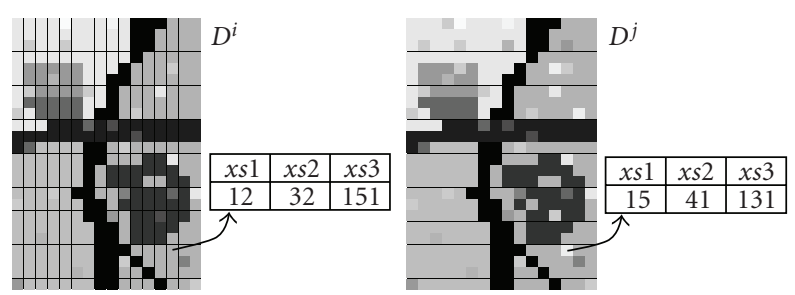

(a) Same resolution/same sensors/different dates: a pixel is described by the same attributes but has different values because of its evolution during the two dates

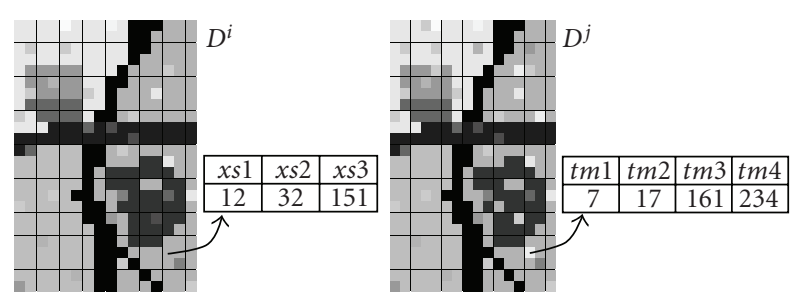

(b) Same resolutions/different sensors: a pixel is described by three attributes in the image on the left, but by four attributes in the image on the right

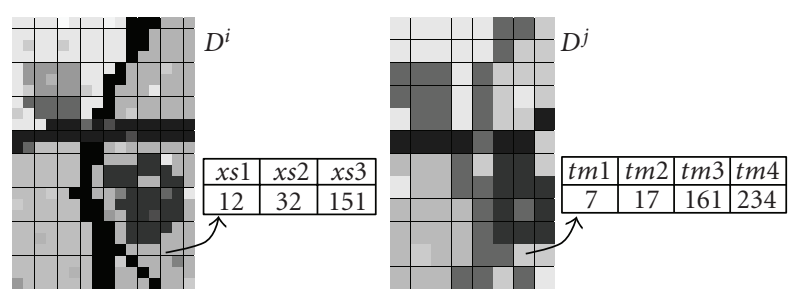

(c) Different resolutions/different sensors: the image $D^{i}$ has a higher resolution than $D^{j}$, the two images do not the same size and the pixels are no more the same

FIgURE 4: The three different cases of image comparison.

curse of dimensionality [16], most of the classical distancebased algorithms are not efficient enough to analyse objects having many attributes, the distances between these objects being not different enough to correctly determine the nearest objects. In addition, the increase of the spectral dimensionality increases the problems like the Hughes phenomena [17] which describes the harmful objects of high-dimensionality objects.

A second way to combine all the attributes (Figure 5(b)) is to first classify the objects with each data sets. These clusterings are made independently. Then a new description of each object is built, using the number of each cluster found by the first classifications. And finally a classification is made using these new descriptions of the objects. The first phase of clusterings enables to reduce the data space for the final clustering, making it easier. This approach is similar to the stacking method [18].

In our approach, the collaborative clustering (Figure $5(c))$ is made quite as in the second method presented above. Each data set is classified according to its attributes. Although the clusterings are not made independently but they are refined to make them converge towards a unique result. Then 


Data $D^{1} \ldots$ Data $D^{N} \longrightarrow$ Clustering $\rightarrow$ Final result

(a) The different data are merged to produce a new dataset which is classified

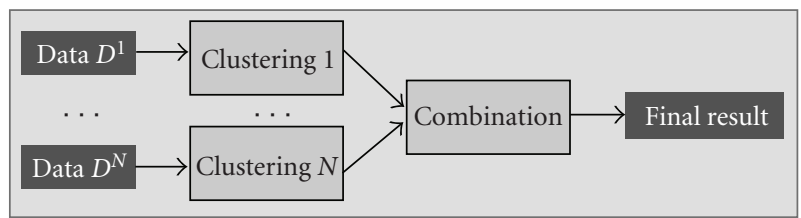

(b) Each dataset is classified independently by a different clustering method and the results are combined

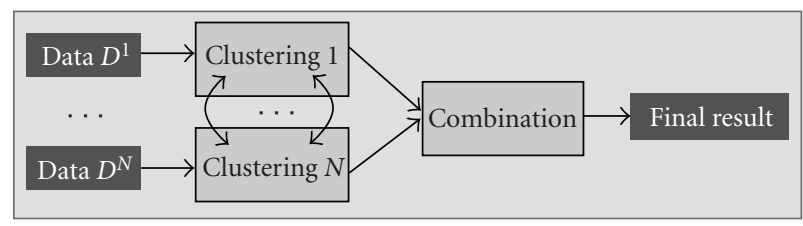

(c) Each dataset is classified by a different clustering method that collaborates with the other methods and then the results are combined

Figure 5: Different data fusion techniques.

only they are unified by a voting method, or a clustering as in method (b).

To integrate this new approach in our system, we affect one dataset to each clustering method. All the process of results refinement stay unchanged, but we are confronted with the problem of the comparison of the different results, and precisely of the estimation of the intercluster similarity (see Section 3.1). In the two first cases presented above (same elements with different descriptions), the confusion matrix and the intercluster similarity defined in Section 3 can be used. However, in the third case (different elements with different descriptions), it cannot be applied because the computation of a confusion matrix between two clusterings involves that the clusters refer to the same objects. The definition of a confusion matrix between datasets of different objects is in the general case very hard, or even impossible. Nevertheless, in some particular problems, it is possible to define it. In the next section, we describe how this matrix can be evaluated in the domain of multiscale remote sensing images clustering.

\subsection{Multiscale remote sensing images classification}

In remote sensing image classification, the problem of the image resolution is not easy to resolve. The resolution of an image is the size covered by one pixel in the real world. For example, the very high-resolution satellites give a resolution of $2.5 \mathrm{~m}$, that is, one pixel is a square of $2.5 \mathrm{~m} \times 2.5 \mathrm{~m}$. One can have different images of a same area but not with the same resolution. So it is really difficult to use these different images because they do not include the same objects to cluster (Figure 6).

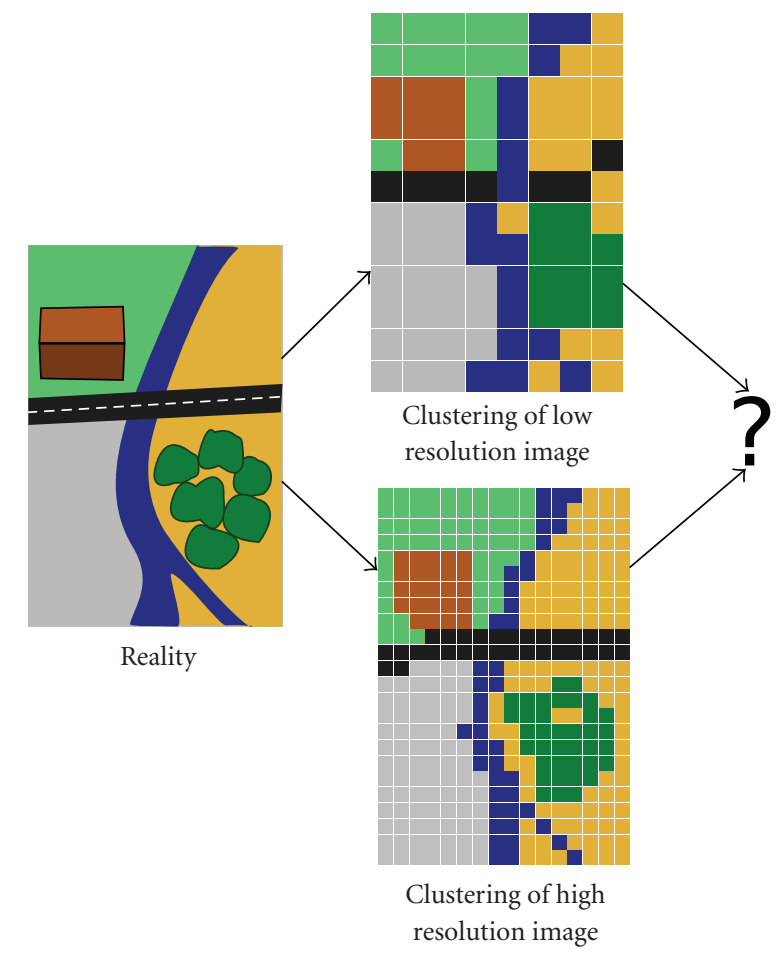

FIGURE 6: How can someone compare objects that are different but that represent a same "real" object? A same reality is viewed at two different resolutions. For example the river is composed of 17 pixels on the low resolution image but it is composed of 43 pixels on the high resolution image.

For example, satellites often produce two kinds of images of the same area, a panchromatic and a multispectral. The panchromatic has a good spatial resolution but a low spectral resolution and, on the contrary, multispectral has a good spectral resolution but a low spatial resolution. A solution to use these two sources of information is to fuse the panchromatic and the multispectral images in a unique one. Many methods have been investigated in the last few years to fuse these two kinds of images and to produce an image with a good spectral and spatial resolution $[19,20]$.

A fused image can be used directly as input of our collaborative system. However, the fused image could not be available or the user would not like to use the fusion or would prefer to process the images without fusing them. In these cases, we have to modify our system to be able to support images at different resolutions. The modification consists of a new definition of the confusion matrix (see (2)) between two clustering results.

In the previous definition given in Section 3, each line of the confusion matrix is given by the confusion vector $\alpha_{k}^{i, j}$ of the cluster $\mathcal{C}_{k}^{i}$ from the result $\mathcal{R}^{i}$ compared to the $n_{j}$ clusters found in the result $\mathcal{R}^{j}$ :

$$
\alpha_{k}^{i, j}=\left(\alpha_{k, l}^{i, j}\right)_{l=1, \ldots, n_{j}}, \quad \text { where } \alpha_{k, l}^{i, j}=\frac{\left|C_{k}^{i} \cap C_{l}^{j}\right|}{\left|C_{k}^{i}\right|} .
$$

If the two results were not computed using the same data and if the resolution of the two images are not the same, it 
is impossible to compute $\left|C_{k}^{i} \cap C_{l}^{j}\right|$. So we propose a new definition of the confusion vector for a class $\mathcal{C}_{k}^{i}$ from the result $\mathcal{R}^{i}$ compared to the result $\mathcal{R}^{j}$.

Definition 1 (new confusion matrix). let $r^{i}$ and $r^{j}$ be the resolution of the two images $I^{i}$ and $I^{j}$; let $\lambda_{I^{1}, I^{2}}$ be a function that associates each pixel of the image $I^{1}$ to one pixel of the image $I^{1}$, with $r^{1} \leq r^{2}$; let $\#\left(\mathcal{C}, I^{1}, I^{2}\right)=\mid\{p \in \mathcal{C}$ : cluster $\left.\left(\lambda_{I^{1}, I^{2}}(p)\right)=\mathcal{C}\right\} \mid ;$ if $r^{i} \leq r^{j}$

$$
\alpha_{k, l}^{i, j}=\frac{\#\left(\mathcal{C}_{k}^{i}, I^{i}, I^{j}\right)}{\left|\mathcal{C}_{k}^{i}\right|}
$$

else

$$
\alpha_{k, l}^{i, j}=\frac{\#\left(\mathcal{C}_{l}^{j}, I^{j}, I^{i}\right)}{\left|\mathcal{C}_{k}^{i}\right|} \times \frac{r^{j}}{r^{i}} .
$$

With this new definition of the confusion matrix, the results can be compared with each other and evaluated as described previously. In the same way, the conflicts resolution phase is unchanged.

Because the images have not the same resolution, it is not possible to apply directly the unification algorithm. In order to build a unique image representing all the results, we choose the maximal resolution and the voting algorithm is applied using the association function $\lambda_{I^{1}, I^{2}}$ for each pixel. This choice was made to produce a result having the best spatial resolution among the different input images.

\section{EXPERIMENTS}

In this section, we present two experiments of our collaborative method on real images. In the first experiment, we use images of the satellite SPOT- 5 to study an urban area. In the second experiment, we use the collaborative method to analyse a coastal zone, through a set of heterogeneous images (SPOT-1, SPOT-5, ASTER).

To be able to use our system with images at different resolutions, we have to define a $\lambda$ function (Figure 7) which defines the correspondence between the pixels of two images. We use here the georeferencing [21] to define this function. In remote sensing, it is possible to associate the real world coordinates to the pixels of an image (i.e., its position on the globe). The georeferencing (here the Lambert 1 North coordinates) is used here to map the pixel from an image to the pixel of another image at a different resolution. By using the georeferencing, we are certain to maximize the quality of the correspondence whatever the difference is between the resolutions of the images.

\subsection{Panchromatic and multispectral collaboration}

The first experiment is the analysis of images of the city of Strasbourg (France). We use the images provided by the sensors of the satellite SPOT-5. The panchromatic image (Figure 8(a)) has a resolution of 5 meters (i.e., the width of one pixel represents 5 meters in the real world), a size of $865 \times 1021$ pixels, and has a unique band. The multispectral

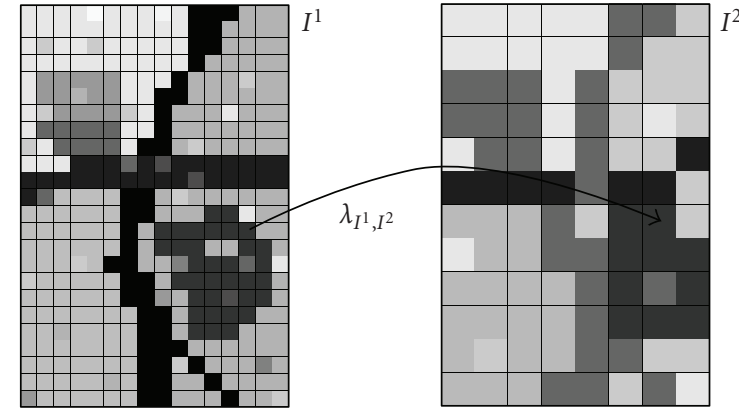

Figure 7: The function $\lambda_{I^{1}, I^{2}}$ is the association function between two images. It enables to associate one pixel of the image $I^{2}$ to each pixel of the image $I^{1}$.

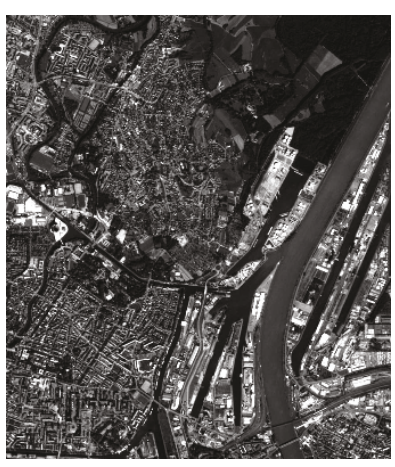

(a) Panchromatic image (resolution 5 meters-size: $865 \times 1021)$

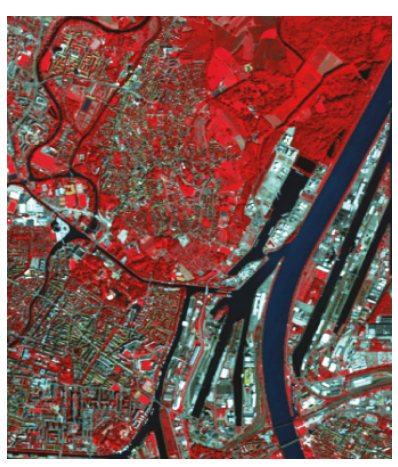

(b) Multispectral image (resolution 10 meters-size: $436 \times 511)$
Figure 8: The two images of Strasbourg (France) from SPOT-5.

image (Figure 8(b)) has a resolution of 10 meters, a size of $436 \times 511$, and has four bands (red, green, blue, and near infrared).

Our goal is to use these two heterogeneous (different resolutions, different number of bands, etc.) sources of data in our collaborative clustering system to show that using multisource images improves the image analysis and scene understanding. Figure 9 presents four different ways to use these two images with our collaborative system:

(a) six clustering methods working on the panchromatic image;

(b) six clustering methods working on the multispectral image;

(c) six clustering methods working on the fusion of the two image;

(d) three clustering methods working on the panchromatic image; and three clustering methods working on the multispectral image.

For case (c), we used the Gram-Schmidt algorithm to merge the panchromatic and the multispectral images. This algorithm is well known in the field of remote sensing image fusion, and produces usually good results [22].

We choose to use the K-Means [23] algorithm for each clustering method. This choice was made for computation 


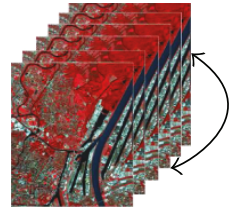

(a) Multispectral: collaborative clustering on the multispectral image

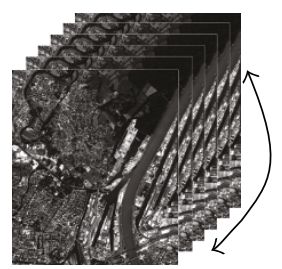

(b) Panchromatic: collaborative clustering on the panchromatic image

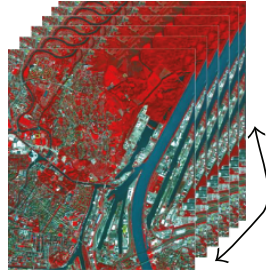

(c) Fusion: collaborative clustering on the fusion of the multispectral and the panchromatic images
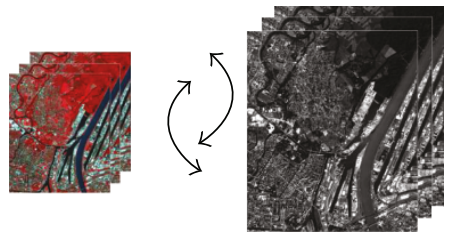

(d) Multisource: multisource collaborative clustering using the panchromatic and the multispectral images

FIGURE 9: The four test cases studied.

TABLE 1: Results with ground truth.

\begin{tabular}{lcccc}
\hline Classes & Multispectral & Panchromatic & Fusion & Collaborative \\
\hline Field 1 & $31.10 \%$ & $24.98 \%$ & $46.12 \%$ & $99.83 \%$ \\
Field 2 & $75.92 \%$ & $67.69 \%$ & $99.23 \%$ & $89.60 \%$ \\
Bridge & $40.74 \%$ & $79.17 \%$ & $35.19 \%$ & $58.80 \%$ \\
Building & $42.24 \%$ & $44.26 \%$ & $67.92 \%$ & $46.42 \%$ \\
\hline Means & $47.50 \%$ & $54.02 \%$ & $62.11 \%$ & $73.66 \%$ \\
\hline
\end{tabular}

convenience, but any clustering method can be used in the collaborative system. For each experiment ((a), (b), (c), and (d)) each clustering method is assigned to one image. Then, the collaborative system described in Section 3 is launched with the modifications added in Section 4 for multiresolution handling, thanks to the georeferencing. The $\mathrm{K}-$ Means algorithm is applied on each image (step 1) with different number of clusters (randomly piked in $[8 ; 10]$ ), and initialized randomly (different initialization for each method). Then, the clustering methods collaborate through the refinement step and modify their results according to the result of the other methods (step 2). Finally, the different results obtained are combined in a single one, thanks to a voting algorithm (step 3). Figure 10 presents the final unification result (obtained from the vote of the different methods) for the four test cases.

All the final results have seven clusters, due to the capacity of the collaborative method to find a consensual number of clusters. According to the interpretation of the geographer expert, the following conclusions can be made. The panchromatic case (Figure 10(b)) has produced a quite bad result where a part of the vegetation has been merged with the water because of the lack of spectral information to describe the pixels (i.e., only one band). The fusion case (Figure 10(c)) has produced a result with a good spatial resolution, but has failed to find some real classes (i.e., the expert expected two clusters of vegetation which have been merged). The multispectral case (Figure 10(a)) has produced a quite good result, but with a low spatial resolution. Finally, the multisource collaboration (Figure 10(d)) has produced a good result with a good spatial resolution, and has corrected some mistakes which appear on the multispectral case. For

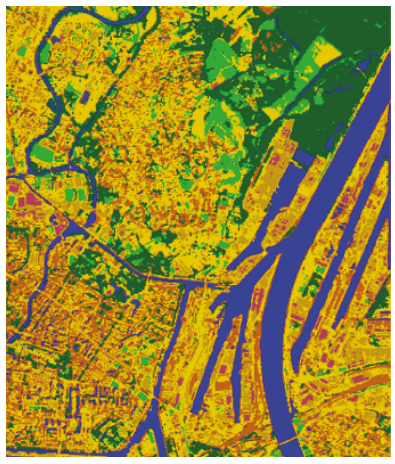

(a) Multispectral (7 clusters)

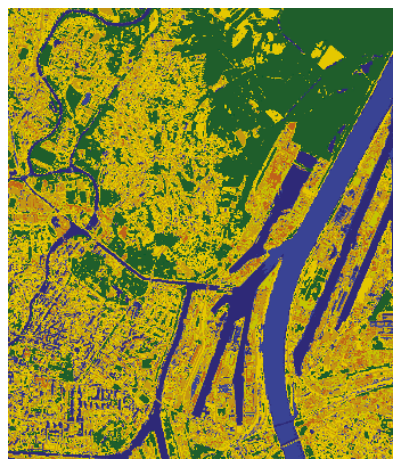

(c) Fusion (7 clusters)

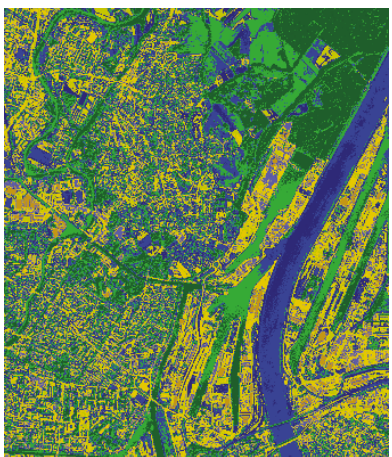

(b) Panchromatic (7 clusters)

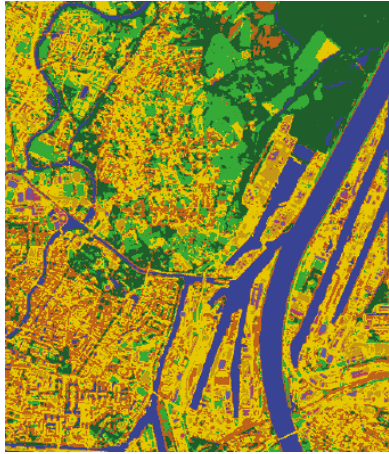

(d) Multisource collaboration (7 clusters)
FIGURE 10: Results for the four test cases studied.

example, the field on the top-right of the area has been identified more precisely thanks to the collaboration with the panchromatic image (Figure 11).

To validate these interpretations, a ground truth has been provided by the expert as partial binaries masks (Figure 11(b)) for four classes. For each ground truth classes, the most potential cluster was selected by the expert (the best overlapping cluster as defined by the Vinet index in [24]). An accuracy index has been computed as the ratio of the number of pixels in the ground truth classes, and the number of pixels of the cluster overlapping it. The results are presented in 


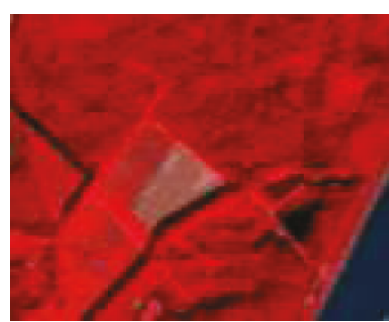

(a) Raw image

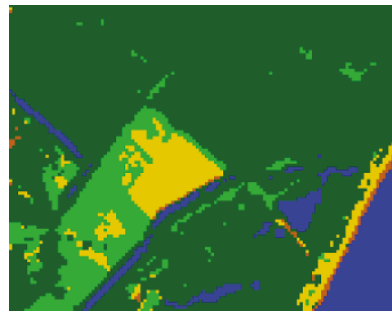

(c) Multispectral

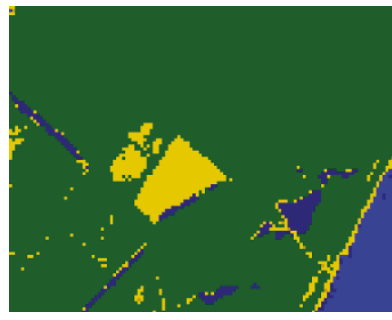

(e) Fusion

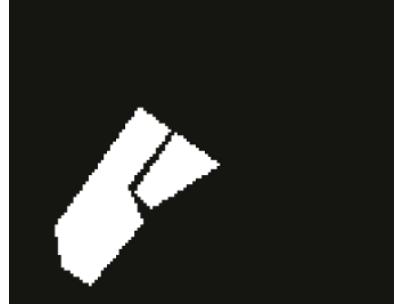

(b) Ground truth

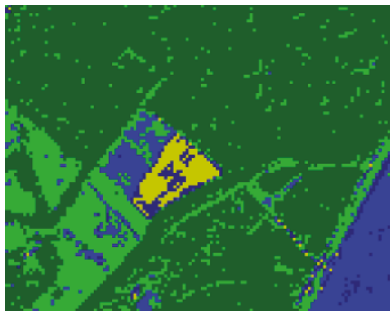

(d) Panchromatic

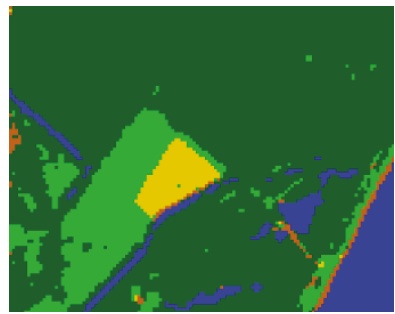

(f) Collaborative
Figure 11: Examples of fields detection. (b) illustrates the ground truth for field (1) (on the left) and field (2) (on the right).

Table 1. As expected, the collaborative solution has produced the best results, especially for the fields detection.

To study the evolution of the agreement amongst all the clustering methods during the refinement step, the tools of the theoretical framework of information theory [25] can be used. random variable. Then, the mutual information [26] can be computed between a couple of clustering results. The mutual information quantify the amount of information shared by the two results. For two results $\mathcal{R}^{i}$ and $\mathcal{R}^{j}$, the $[0 ; 1]$ normalized mutual information is defined as

$$
\operatorname{nmi}\left(\mathcal{R}^{i}, \mathcal{R}^{j}\right)=\frac{2}{p} \sum_{k=1}^{n_{i}} \sum_{l=1}^{n_{j}} \log _{n_{i} \cdot n_{j}}\left(\frac{p \cdot \alpha_{k, l}^{i, j}}{n_{k}^{i} \cdot n_{l}^{j}}\right),
$$

where $p$ is the number of pixels to classify, $n_{i}$ is the number of clusters from $\mathcal{R}^{i}$, and $n_{k}^{i}$ is the number of objects in the cluster $\mathcal{C}_{k}^{i}$ from $\mathcal{R}^{i}$.

Moreover, the average mutual information quantify the shared information among an ensemble of clustering results, and can be used as an indicator of agreement:

$$
\operatorname{anmi}(m)=\frac{1}{N-1} \sum_{j=1, j \neq m}^{N} \operatorname{nmi}\left(\mathcal{R}^{m}, \mathcal{R}^{j}\right)
$$

with $m=1,2, \ldots, N$, and $N$ the number of clustering results.

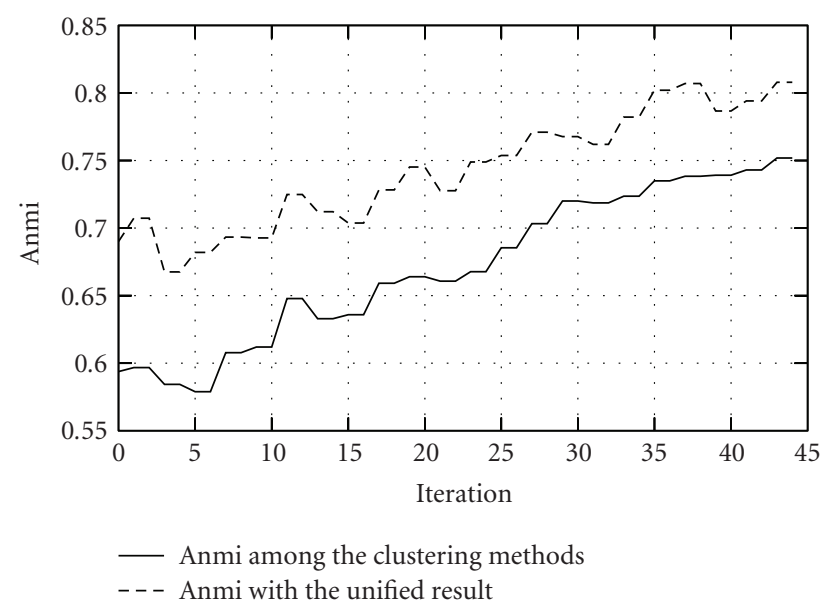

FIGURE 12: Evolution of the anmi index among the clustering methods and the average nmi between the results and the unified result.

The average mutual information has been computed during the refinement process which have produced the result of Figure 10(d). Figure 12 presents the evolution of the anmi index among the results of the different clustering methods, and the average of the mutual information between each clustering method and the unified result.

\subsection{Multiresolution multidate collaboration}

The second experiment was made on four images of a coastal zone (Normandy Coast, Northwest of France). This area is very interesting because it is periodically affected by natural and anthropic phenomena which modify the structure of the area. Consequently, the expert has often a lot of heterogeneous images available which are acquired through the years. Four images issued from three different satellites (SPOT-4, SPOT-5 and ASTER) and having different resolutions $(20,15,10$, and 2.5 meters) are used.

Four clustering methods were set up, each one using one of the available images. As in the previous experiment, the K-Means algorithm is ran on each image (step 1), the refinement algorithm is then applied (step 2), and the results are combined (step 3). Figure 14 presents the result of the unification of the final results.

To make a better interpretation of the unified result, a vote map is produced. This map represents the result of the vote carried out during the combination of the results [15]. Figure 15 presents the vote map corresponding to the result shown in Figure 14. In this image, the darker the pixels are, the less the clustering methods are in agreement. So, the pixels where all the clustering methods agreed are in white, and the black pixels represent a strong disagreement amongst the clustering methods. This degree of agreement is computed using the corresponding cluster (see (1)). This representation helps the expert to improve his analysis of the result, by concentrating his attention on the part of the image where the clustering methods are in disagreement. 


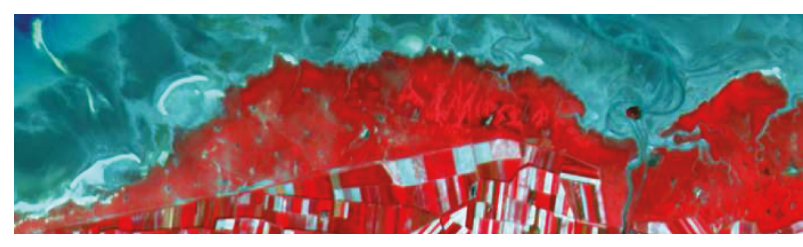

(a) SPOT-4-20 meters-3 bands $(659 \times 188)$-date: 1999

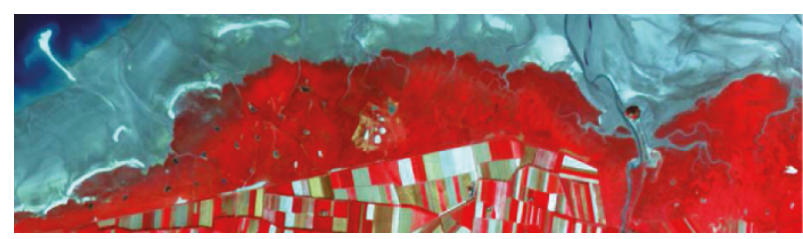

(b) ASTER-15 meters-3 bands $(922 \times 256)$-date: 2004

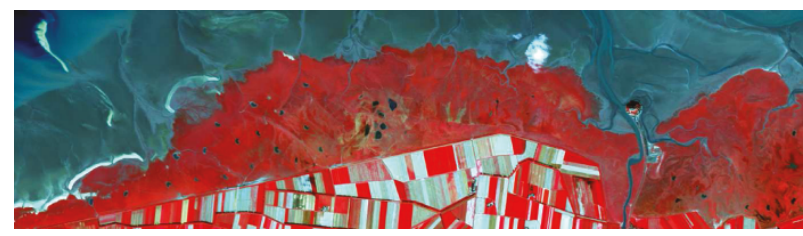

(c) SPOT-4-10 meters-3 bands $(1382 \times 384)$-date: 2002

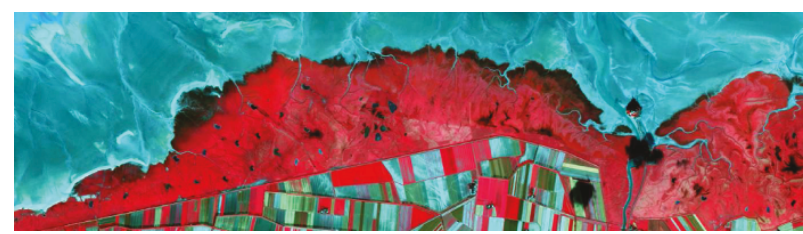

(d) SPOT-5-2.5 meters-3 bands $(5528 \times 1536)$-date: 2005

FIgure 13: The four images of Normandy Coast, France.

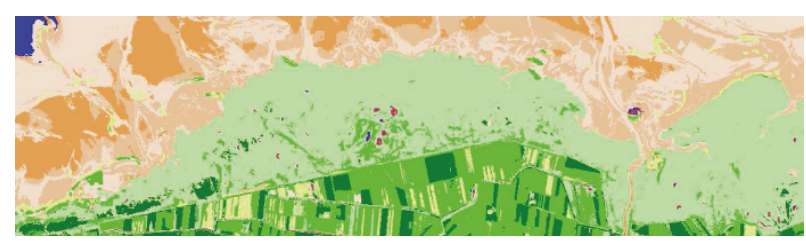

FIgUre 14: The final unification result.

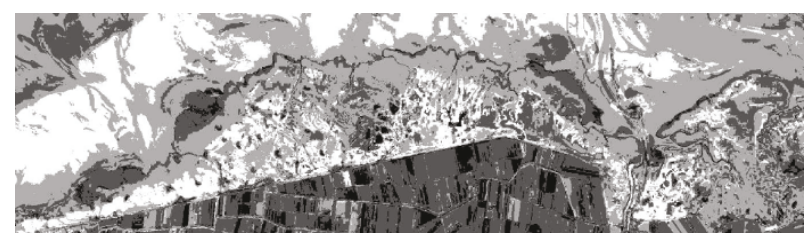

Figure 15: The vote map.

Consequently, another way to improve the scene understanding and to show the agreement between the methods is to visualise the corresponding clusters (1) between a pair of results. It allows the expert to see which parts of the clusters are in agreement, and which parts are in disagreement, for a couple of results. Figure 16 presents two corresponding clusters between the clustering methods of this experiment.

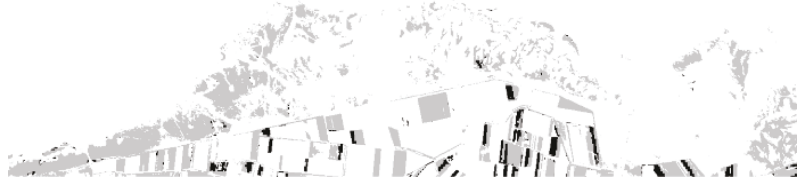

(a) Corresponding clusters showing disagreement in the fields

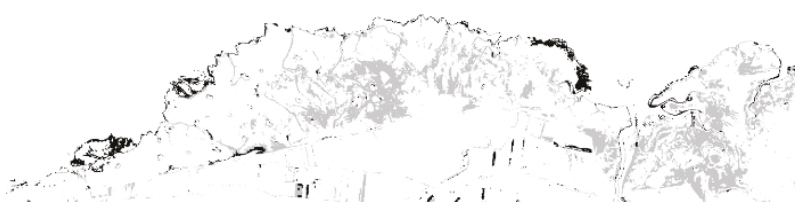

(b) Corresponding clusters showing a part of the coast line

FIGURE 16: Corresponding clusters between two clustering methods, in grey the agreement, in black the disagreement.

In Figure 16(a), one can see the disagreement on a part of the coast line. Figure 16(b) illustrates the disagreement on the fields. All these results help the expert to improve his image understanding.

\section{CONCLUSIONS}

In this paper, we have presented a method of multisource images analysis using collaborative clustering. This collaborative method enables the user to exploit different heterogeneous images in an overall system. Each clustering method works on one image and collaborates with the other clustering methods to refine its result.

Experimentations for the analysis of an urban area and a coastal area have been presented. The system produces a final result by combining the results of the different clustering methods using a voting algorithm. The agreement and the disagreement of the clustering methods can be highlighted by a vote map, depicting the accordance between the different clustering methods. Furthermore, the corresponding clusters between a pair of clustering methods can be visualised. These features are very useful to help the expert to better understand his images.

However, there is still a lot of work for the expert to really interpret the information in the dataset because no semantic is given by the system. That is why we are working on an extension of this process, integrating highlevel domain knowledge on the studied area (urban objects ontology, spatial relationships, etc.). This should enable to add automatically semantic to the result, giving more information to the user.

\section{ACKNOWLEDGMENTS}

The authors would like to thank the members of the FodoMuST and Ecosgil projects for providing the images and the geographers of the LIV Laboratory for their help in the interpretation of the results. This work is supported by the french Centre National d'Etudes Spatiales (CNES Contract 70904/00). 


\section{REFERENCES}

[1] T. M. Mitchell, Machine Learning, McGraw-Hill, New York, NY, USA, 1997.

[2] A. K. Jain, M. N. Murty, and P. J. Flynn, "Data clustering: a review," ACM Computing Surveys, vol. 31, no. 3, pp. 264-323, 1999.

[3] C. Pohl and J. L. Van Genderen, "Multisensor image fusion in remote sensing: concepts, methods and applications," International Journal of Remote Sensing, vol. 19, no. 5, pp. 823854, 1998.

[4] Y. Chibani, "Selective synthetic aperture radar and panchromatic image fusion by using the à trous wavelet decomposition," EURASIP Journal on Applied Signal Processing, vol. 2005, no. 14, pp. 2207-2214, 2005.

[5] Y.-L. Chang, L.-S. Liang, C.-C. Han, J.-P. Fang, W.-Y. Liang, and K.-S. Chen, "Multisource data fusion for landslide classification using generalized positive boolean functions," IEEE Transactions on Geoscience and Remote Sensing, vol. 45, no. 6, pp. 1697-1708, 2007.

[6] M.-P. Dubuisson and A. K. Jain, "Contour extraction of moving objects in complex outdoor scenes," International Journal of Computer Vision, vol. 14, no. 1, pp. 83-105, 1995.

[7] M. Germain, M. Voorons, J.-M. Boucher, G. B. Bénié, and E. Beaudry, "Multisource image fusion algorithm based on a new evidential reasoning approach," ISPRS Journal of Photogrammetry \& Remote Sensing, vol. 35, part 7, pp. 1263-1267, 2004.

[8] J. A. Benediktsson and I. Kanellopoulos, "Classification of multisource and hyperspectral data based on decision fusion," IEEE Transactions on Geoscience and Remote Sensing, vol. 37, no. 3, pp. 1367-1377, 1999.

[9] L. Bruzzone, R. Cossu, and G. Vernazza, "Combining parametric and non-parametric algorithms for a partially unsupervised classification of multitemporal remote-sensing images," Information Fusion, vol. 3, no. 4, pp. 289-297, 2002.

[10] M. Fauvel, J. Chanussot, and J. A. Benediktsson, "Decision fusion for the classification of urban remote sensing images," IEEE Transactions on Geoscience and Remote Sensing, vol. 44, no. 10, part 1, pp. 2828-2838, 2006.

[11] A. Gionis, H. Mannila, and P. Tsaparas, "Clustering aggregation," in Proceedings of the 21st International Conference on Data Engineering (ICDE '05), pp. 341-352, Tokyo, Japan, April 2005.

[12] A. L. N. Fred and A. K. Jain, "Combining multiple clusterings using evidence accumulation," IEEE Transactions on Pattern Analysis and Machine Intelligence, vol. 27, no. 6, pp. 835-850, 2005.

[13] A. Strehl and J. Ghosh, "Cluster ensembles-a knowledge reuse framework for combining multiple partitions," Journal of Machine Learning Research, vol. 3, no. 3, pp. 583-617, 2003.

[14] Z.-H. Zhou and W. Tang, "Clusterer ensemble," KnowledgeBased Systems, vol. 19, no. 1, pp. 77-83, 2006.

[15] C. Wemmert and P. Gançarski, "A multi-view voting method to combine unsupervised classifications," in Proceedings of the 2nd IASTED International Conference on Artificial Intelligence and Applications (AIA '02), pp. 362-324, Malaga, Spain, September 2002.

[16] R. E. Bellman, Adaptive Control Processes, Princeton University Press, Princeton, NJ, USA, 1961.

[17] G. F. Hughes, "On the mean accuracy of statistical pattern recognizers," IEEE Transactions on Informations Theory, vol. 14, no. 1, pp. 55-63, 1968.

[18] L. I. Kuncheva, Combining Pattern Classifiers: Methods and Algorithms, Wiley-Interscience, New York, NY, USA, 2004.
[19] W. Dou, Y. Chen, X. Li, and D. Z. Sui, “A general framework for component substitution image fusion: an implementation using the fast image fusion method," Computers \& Geosciences, vol. 33, no. 2, pp. 219-228, 2007.

[20] V. Karathanassi, P. Kolokousis, and S. Ioannidou, "A comparison study on fusion methods using evaluation indicators," International Journal of Remote Sensing, vol. 28, no. 10, pp. 2309-2341, 2007.

[21] L. L. Hill, Georeferencing: The Geographic Associations of Information, Digital Libraries and Electronic Publishing, The MIT Press, Cambridge, Mass, USA, 2006.

[22] C. Li, L. Liu, J. Wang, C. Zhao, and R. Wang, "Comparison of two methods of the fusion of remote sensing images with fidelity of spectral information," in Proceedings of the IEEE International Geoscience and Remote Sensing Symposium (IGARSS '04), vol. 4, pp. 2561-2564, Anchorage, Alaska, USA, September 2004.

[23] J. McQueen, "Some methods for classification and analysis of multivariate observations," in Proceedings of the 5th Berkeley Symposium on Mathematical Statistics and Probability, vol. 1, pp. 281-297, Berkeley, Calif, USA, June-July 1967.

[24] S. Chabrier, B. Emile, C. Rosenberger, and H. Laurent, "Unsupervised performance evaluation of image segmentation," EURASIP Journal on Applied Signal Processing, vol. 2006, Article ID 96306, 12 pages, 2006.

[25] T. M. Cover and J. A. Thomas, Elements of Information Theory, Wiley-Interscience, New York, NY, USA, 1991.

[26] A. Strehl, "Relationship-based clustering and cluster ensembles for high-dimensional data mining," Ph.D. thesis, The University of Texas at Austin, Austin, Tex, USA, May 2002. 